\title{
Vector Extrapolation-based Acceleration of Regularized Richardson Lucy Image Deblurring
}

\author{
Steffen Remmele ${ }^{1}$, Jürgen Hesser ${ }^{1,2}$ \\ ${ }^{1}$ Institute for Computational Medicine, University of Heidelberg \\ ${ }^{2}$ Experimental Radiooncology, Medical Centre Mannheim, University of Heidelberg \\ steffen.remmele@ziti.uni-heidelberg.de
}

\begin{abstract}
Confocal fluorescence microscopy has become an important tool in biological and medical sciences for imaging thin specimen, even living ones. Due to out-of-focus blurring and noise the acquired images are degraded and thus it is necessary to restore them. One of the most popular methods is an iterative Richardson-Lucy algorithm with total variation regularization. This algorithm while improving the image quality is converging slowly whereas with a constantly increasing amount of image data fast methods are required. In this paper, we present an accelerated version of the algorithm and investigate the achieved speed up. The acceleration method is based on a vector extrapolation technique and avoids a computational intensive evaluation of the underlying cost function. To evaluate the acceleration two synthetic test images are used. The accelerated algorithm reaches an acceptable result within $30 \%$ to $40 \%$ less computational time.
\end{abstract}

\section{Introduction}

\subsection{Image formation model}

Confocal fluorescence microscopy images suffer from both blurring and Poisson noise. The images are blurred due to the optical aperture and the physical resolution of light itself. This blurring is described by a convolution with a point spread function (PSF) [1]. In this paper, the PSF is noted as $h$ and we assume that it is known. In addition, since confocal microscopy is a low-photon imaging technique the acquired images are degraded by Poisson noise $\phi($.$) . Thus$ a suitable image formation model, where $i$ is the observed image, $o$ the original image and $\otimes$ represents the convolution operator is given by:

$$
i=\phi(o \otimes h)
$$

The likelihood of an original image $o$ for a recorded image $i$ is given by the following equation due to the Poisson process describing the low-photon imaging nature of the confocal fluorescence microscopy. In this distribution, it is assumed that the noise is statistically uncorrelated.

$$
P(i \mid o)=\prod_{x \in \Omega} \frac{[(o \otimes h)(x)]^{i(x)} e^{-(o \otimes h)(x)}}{i(x) !}
$$




\subsection{Deblurring methods}

Maximizing the probability $P(i \mid o)$ with respect to $o$ using a multiplicative gradient-based algorithm (expectation-maximization) leads to the Richardson-Lucy (RL) algorithm [2], [3]:

$$
o_{k+1}(x)=\left\{\left[\frac{i(x)}{\left(o_{k} \otimes h\right)(x)}\right] \otimes h(-x)\right\} o_{k}(x)
$$

The RL algorithm improves the image quality first but after several iterations noise is amplified and the algorithm does not converge. In order to overcome this problem a priori knowledge $P(o)$ about the image is added and the a posteriori distribution $P(o \mid i)=P(i \mid o) P(o)$ is then maximized (MAP). The a priori model is included by adding a regularization term and in the last decade several regularization terms have been introduced. In [4] a Total Variation (TV) regularization suppresses noise amplification by smoothing the image while at the same time preserving the edges. Introduced in [5], TV was used to denoise images. A TV regularized multiplicative RL algorithm (RLTV) with $\lambda$ being the regularization parameter and $\nabla$ being the gradient is given by:

$$
o_{k+1}(x)=\left\{\left[\frac{i(x)}{\left(o_{k} \otimes h\right)(x)}\right] \otimes h(-x)\right\} \frac{o_{k}(x)}{1-\lambda \nabla \frac{\nabla o_{k}(x)}{\left|\nabla o_{k}(x)\right|}}
$$

\subsection{Acceleration of RL algorithm}

In [6], an adaptive accelerated Richardson-Lucy method is presented. The main objective of this work is the acceleration of the convergence speed of the standard multiplicative RL algorithm presented in (3) by adding an exponent to the multiplicative correction ratio. This exponenet is computed adaptively by using the deblurred images from previous iteration steps. Since there is no regularization in this method, noise amplification is a problem. Supervising the root mean square error (RMSE) shows a considerable noise amplification after few iterations. Thus, the authors suggest stopping the algorithm at the minimum RMSE. In case of noise-free images, it is shown that the new accelerated methods is able to obtain better results regarding the RMSE after less iterations.

\section{Materials and methods}

\subsection{Acceleration of RLTV}

In order to accelerate the multiplicative RLTV (accRLTV) algorithm (4), we use a method introduced by Biggs and Andrews [7]. This acceleration method is based on vector extrapolation and does not require an evaluation of the cost function. In general, the method can be applied to various techniques as long as the basic iterative algorithm contains a known independent functional $\psi$ that 
can be used to calculate the next iteration step (5). The multiplicative RLTV fulfills this requirement.

$$
o_{k+1}=\psi\left(o_{k}\right)
$$

In the original algorithm the current iteration $o_{k}$ is used in the functional $\psi$ in order to get the next iteration $o_{k+1}$. In the accelerated version, a virtual point $g_{k}$ is calculated and this point is used in the functional $\psi$ to determine the next iteration $o_{k+1}=\psi\left(g_{k}\right)$. In order to determine the virtual point $g_{k}$, the direction $d_{k}=o_{k}-o_{k-1}$ being the difference between the current and the previous iteration is used. In addition, an acceleration parameter $\alpha_{k}$ is needed to weight the direction $d_{k}$ when calculating the virtual point $g_{k}$ :

$$
g_{k}=o_{k}+\alpha_{k} \cdot d_{k}
$$

In the used method, $\alpha_{k}$ is estimated by using the results of previous iteration steps. A more accurate solution could be obtained by a line search but this is no option because of the computational effort. In order to estimate $\alpha_{k}$, the differences $l_{k}$ between the virtual point $g_{k-1}$ and the respectively next iteration $o_{k}$ are used $l_{k}=o_{k}-g_{k-1}$. These differences describe the actual changes made by the functional $\psi . \quad \alpha_{k}$ is then calculated using following formula where $\odot$ denotes an element-wise multipication and $\sum$ sums all elements in the data.

$$
\alpha_{k}=\frac{\sum_{\Omega}\left(l_{k-1} \odot l_{k-2}\right)}{\sum_{\Omega}\left(l_{k-1} \odot l_{k-1}\right)}
$$

In addition, each $\alpha$ has to fulfill the condition $0 \leq \alpha \leq 1$ and is modified after the estimation accordingly in case this condition is not fulfilled. Figure 1 shows graphically how the acceleration influences the calculation. It can be seen that instead of the original step size much larger changes can be achieved in each iteration step by using the virtual points.

\subsection{Numerical stability}

The values in the fluorescence images are positive or zero since they represent a photon count. The RLTV (4), however, is not well defined for zero values and thus a modified version of the TV regularization is applied [8]. Additionally, the values used for $\lambda$ are restricted in order to avoid negative or zero values in the denominator and we thus use 0.002 as suggested in [4].

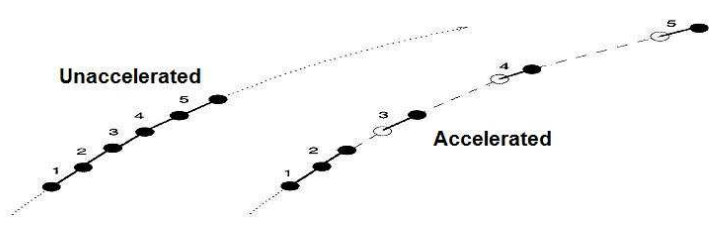

Fig. 1. Illustration of acceleration 
Fig. 2. left to right: original image, blurred and noisy image, RLTV, accRLTV
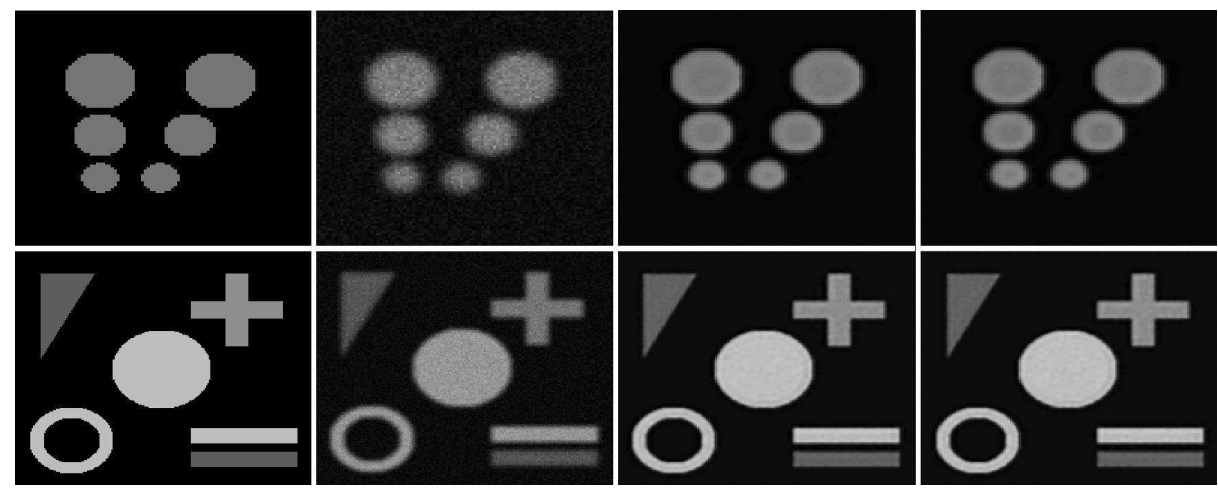

\subsection{Quality of the results}

To quantify the quality of the restored images, it is usual to use the I-divergence (IDiv) being equivalent to the likelihood distribution of the Poisson noise (2). IDiv compares the recorded image $i$ with the reblurred reconstructed image $o \otimes h$. Since the original image $\tilde{o}$ is known for our synthetic test images, we use the mean square error (MSE) to compare $\tilde{o}$ with the reconstructed image $o$ as well.

$$
\operatorname{Idiv}_{A, B}=\sum_{x \in \Omega}\left\{A(x) \ln \left(\frac{A(x)}{B(x)}\right)-(A(x)-B(x))\right\}
$$

\section{Results}

For our evaluation, we use two synthetic test images $(3 \mathrm{D})$ sized $100 \times 100 \times 60$ voxels and $200 \times 200 \times 50$ voxels. The images are blurred with a Gaussian PSF of size $9 \times 9 \times 9$ voxels with a variance of 1.8 voxel size and corrupted by Poisson noise. One slice of each test image together with their degraded versions are shown in figure (2). Furthermore, the results of the deblurring for the RLTV and accRLTV are displayed.

In average, one iteration of the accRLTV algorithm takes $5 \%$ longer than the RLTV (Average time for one iteration image 1: RLTV 1.38 seconds and
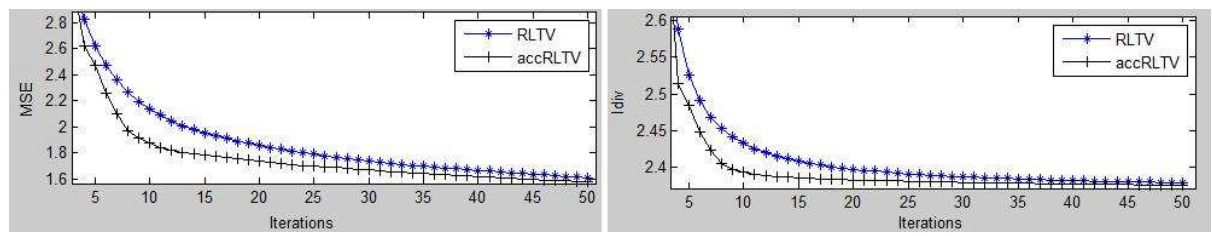

Fig. 3. a) MSE b) IDiv 
Table 1. Amount of iterations to deblur the images

\begin{tabular}{lcccccc}
\hline & \multicolumn{7}{c}{ RLTV(1) } & accRLTV(1) & RLTV(2) & accRLTV(2) & RLTV(3) & accRLTV(3) \\
\hline first test image & 29 & 18 & 31 & 21 & 30 & 19 \\
second test image & 25 & 15 & 25 & 14 & 26 & 16 \\
\hline
\end{tabular}

accRLTV 1.45 seconds with a $2.6 \mathrm{GHz}$ Quad Core CPU). Each test image was degraded three times and restored with both the RLTV and the accRLTV. The deblurring is stopped when a certain MSE is reached and the amount of necessary iterations is shown in Table 1. The progress of both the MSE and the IDiv are shown in Figure 3 for one test image.

\section{Discussion}

In Figure 2, it can be seen that the result images of both algorithms are nearly identical. Since the differences in the computational time for both algorithms are quite small the computational time can be neglected in the further evaluation. For the first test image the desired MSE is reached by the accRLTV within 30\% less iterations compared with the RLTV. In case of the second test image the improvement is even higher: it takes $40 \%$ less iterations to reach the specified MSE for the accRLTV. The progress of the MSE and Idiv in figure 3 shows graphically that the accRLTV reconstructs the image faster than the original algorithm. In summary, the accRLTV produces acceptable results and requires considerably less computational time than the RLTV.

\section{References}

1. Yoo H, Song I, Gweon DG. Measurement and restoration of the point spread function of fluorescence confocal microscopy. J Microscopy. 2005;221:172-176.

2. Lucy LB. An iterative technique for rectification of observed distributions. Astronom J. 1974; 79:745-765.

3. Richardson WH. Bayesian-based iterative method of image restoration. JOSA. 1972;62:55-59.

4. Dey N, Blanc-Féraud L, Zimmer C, et al. Richardson-Lucy algorithm with total variation regularization for $3 \mathrm{D}$ microscope deconvolution. Microscope Res Techn. 2006;69:260-266.

5. Rudin LI, Osher S, Fatemi E. Nonlinear total variation based noise removal algorithms. Physica D. 1992;60:259-268.

6. Singh MK, Tiwary US, Kim YH. An adaptively accelerated Lucy-Richardson method for image deblurring. EURASIP J Adv Signal Process. 2008;8:1-10.

7. Biggs D, Andrews M. Acceleration of iterative image restoration algorithms. Appl Optics. 1997;36:123-145.

8. Chan T, Marquina A, Mulet P. High-order total variation-based image restoration. SIAM J Sci Comput. 2000;22:503-516. 